\title{
Avaliação de métodos para estimativa da evapotranspiração de referência no estado do Piauí
}

\author{
Aderson Soares de Andrade Junior ${ }^{1(*)}$, Clescy Oliveira da Silva ${ }^{2}$, Valdemício Ferreira de Sousa ${ }^{3}$, Valdenir Queiroz Ribeiro ${ }^{4}$ \\ ${ }^{1}$ Pesquisador, Embrapa Meio-Norte, Teresina, PI, aderson.andrade@embrapa.br \\ ${ }^{2}$ Mestre em Engenharia Agrícola, UFC, Fortaleza, CE, clescy@gmail.com \\ ${ }^{3}$ Pesquisador, Embrapa Meio-Norte, Teresina, PI, valdemicio.sousa@embrapa.br \\ ${ }^{4}$ Pesquisador, Embrapa Meio-Norte, Teresina, PI, valdenir.queiroz@embrapa.br \\ ${ }^{(*)}$ Autor para correspondência
}

\section{INFORMAÇÕES}

\section{História do artigo:}

Recebido em 16 de Junho de 2017

Aceito em 10 de agosto de 2017

\section{Termos para indexação:}

equações empíricas

índice de confiança

Índice de Willmott

\section{RESUMO}

O estudo objetivou estimar a evapotranspiração de referência (ETo) por métodos que utilizam menor número de variáveis meteorológicas, comparando-os com o método padrão Penman-Monteith. Os dados meteorológicos foram fornecidos pelo Instituto Nacional de Meteorologia (INMET) de 19 estações automáticas localizadas no Estado do Piauí. A estimativa da ETo foi feita para os períodos seco e chuvoso do ano, pelos métodos de Hargreaves \& Samani (HS), Priestley-Taylor (PT), Thornthwaite (TH), Thornthwaite com temperatura efetiva (THtef) e Penman-Monteith FAO (PM-FAO). O estudo foi modelado utilizando-se o delineamento inteiramente casualizado para cada estação meteorológica automática. Os métodos estatísticos utilizados na análise foram: teste de Tukey ao nível de 5\% de probabilidade (para as médias de ETo), correlação de Pearson, teste de Fisher, regressão linear, coeficiente de Willmott e índice de confiança ou desempenho. O método de PT apresentou desempenho de "bom" a "ótimo" em quase todos os municípios avaliados, notadamente, no período chuvoso. O método de TH não apresentou desempenho satisfatório com relação a PM-FAO. A adoção da correção da estimativa de ETo por TH pelo emprego da temperatura efetiva (THtef) eleva o nível de desempenho das estimativas de ETo com relação a PM-FAO.

(c) 2017 SBAgro. Todos os direitos reservados.

\section{Introdução}

O setor que mais consome água é o agropecuário, cerca de $80 \%$, sendo que $69 \%$ são destinados a irrigação. No Nordeste brasileiro, a escassez de água é muito maior que no restante do país; portanto, para o desenvolvimento do setor agrícola é necessária aplicação de tecnologias e planejamento que venha a tornar a irrigação na região mais eficiente, utilizando água de maneira racional. A irrigação deve ser feita de maneira a suprir a necessidade da planta sem desperdício.

A necessidade da planta por água é baseada no cálculo da evapotranspiração, que corresponde à perda de água pela planta por transpiração e pela evaporação do solo. A evapotranspiração pode ser estimada por vários métodos empíricos, sendo que o método recomendado pela FAO é o método Penman-Monteith (ALLEN et al., 1998), que envolve um número grande de variáveis como: umidade relativa 
do ar, radiação solar, temperatura do ar e velocidade do vento.

Contudo, são poucos os locais que dispõem de dados meteorológicos completos para estimativa da ETo. Na maioria das vezes se encontram incompletos ou indisponíveis para serem utilizados nos cálculos em método que requer maior número de informações, como é caso de Penman-Monteith. Por isso, torna-se necessário fazerem-se avaliações de métodos alternativos e mais simplificados para estimativa da ETo visando a sua futura orientação de uso.

Dessa forma, este estudo tem como objetivo estimar a evapotranspiração de referência (ETo) por métodos que utilizam menor número de variáveis meteorológicas, tais como os de Priestley-Taylor, Hargreaves \& Samani e Thornthwaite, comparando-os com o método padrão Penman-Monteith, para alguns municípios do Estado do Piauí.

\section{Material e métodos}

Para a realização do estudo utilizaram-se dados de 19 estações meteorológicas automáticas em operação, pertencentes à rede de estações do Instituto Nacional de Meteorologia (INMET), localizadas no Estado do Piauí. As variáveis climáticas coletadas pelas estações, em escala diária, são: temperatura do ar, radiação solar global, umidade relativa do ar, velocidade do vento, pressão atmosférica e precipitação pluvial. o período de coleta dos dados para cada estação variou de 2008 a 2011. A localização geográfica das estações, bem como as coordenadas e período de coleta de dados podem ser visualizadas na Tabela 1 e Figura 1. Os dados foram analisados para os períodos seco e chuvoso do ano. Considerou-se como período chuvoso, os meses de dezembro a abril, e como período seco, os meses de maio a novembro.

Para se estimar a ETo consideraram-se os seguintes métodos: Penman-Monteith FAO (PM-FAO) (ALLEN et al., 1998), Priestley-Taylor (PT) (PRISESTLEY, TAYLOR, 1972), Hargreaves \& Samani (HS) (HARGREAVES, SAMANI, 1985), Thornthwaite (TH) (THORNTHWAITE, 1948) e Thornthwaite com a temperatura efetiva (THtef) (CAMARGO et al., 1999).

Modelou-se o estudo usando-se o delineamento experimental inteiramente casualizado para cada estação climatológica automática e as médias foram comparadas pelo teste "t" ao nível de $5 \%$ de probabilidade. Para se estimar o grau de diferença entre o método PM-FAO e os outros

Tabela 1. Estações, coordenadas geográficas, período e número de dias por ano com dados climáticos nas estações meteorológicas utilizadas no estudo.

\begin{tabular}{|c|c|c|c|c|c|c|c|c|c|}
\hline \multirow{2}{*}{ N. } & \multicolumn{3}{|c|}{ Coordenadas Geográficas } & \multirow{2}{*}{ Período } & \multicolumn{5}{|c|}{ Número de dias com dados } \\
\hline & Lat & Long & Alt & & 2008 & 2009 & 2010 & 2011 & Total \\
\hline 1 Alvorada do Gurguéia & $-8,44^{\circ}$ & $-43,86^{\circ}$ & 270 & $2008-2011$ & 334 & 338 & 333 & 151 & 1156 \\
\hline 2 Bom Jesus & $-9,08^{\circ}$ & $-44,33^{\circ}$ & 297 & $2008-2011$ & 333 & 284 & 259 & 149 & 1025 \\
\hline 3 Canto do Buriti & $-8,12^{\circ}$ & $-42,97^{\circ}$ & 308 & $2010-2011$ & & & 155 & 179 & 334 \\
\hline 4 Caracol & $-9,29^{\circ}$ & $-43,32^{\circ}$ & 100 & $2008-2011$ & 273 & 272 & 247 & 180 & 972 \\
\hline 5 Castelo do Piauí & $-5,35^{\circ}$ & $-41,51^{\circ}$ & 286 & 2009-2010 & & 255 & 54 & & 309 \\
\hline 6 Esperantina & $-3,90^{\circ}$ & $-42,26^{\circ}$ & 65 & $2008-2011$ & 362 & 254 & 220 & 32 & 868 \\
\hline 7 Floriano & $-6,77^{\circ}$ & $-43,02^{\circ}$ & 123,3 & $2008-2011$ & 324 & 305 & 331 & 150 & 1110 \\
\hline 8 Gilbués & $-9,87^{\circ}$ & $-45,35^{\circ}$ & 425 & 2010-2011 & & & 216 & 180 & 396 \\
\hline 9 Oeiras & $-6,97^{\circ}$ & $-42,15^{\circ}$ & 156 & $2008-2011$ & 163 & 329 & 332 & 136 & 960 \\
\hline 10 Parnaíba & $-3,07^{\circ}$ & $-41,78^{\circ}$ & 57 & $2008-2011$ & 304 & 173 & 330 & 123 & 930 \\
\hline 11 Paulistana & $-8,13^{\circ}$ & $-41,14^{\circ}$ & 374 & $2008-2011$ & 365 & 352 & 303 & 120 & 1140 \\
\hline 12 Picos & $-7,07^{\circ}$ & $-41,40^{\circ}$ & 233 & $2008-2011$ & 16 & 288 & 280 & 38 & 622 \\
\hline 13 Piripiri & $-4,28^{\circ}$ & $-41,79^{\circ}$ & 161 & $2008-2010$ & 284 & 351 & 302 & 60 & 997 \\
\hline 14 São João do Piauí & $-8,36^{\circ}$ & $-42,25^{\circ}$ & 235 & $2008-2011$ & 365 & 287 & 303 & 148 & 1103 \\
\hline 15 São Pedro do Piauí & $-5,91^{\circ}$ & $-42,72^{\circ}$ & 287 & 2009-2011 & 79 & 2008 & 149 & 178 & 2414 \\
\hline 16 São Raimundo Nonato & $-9,03^{\circ}$ & $-42,70^{\circ}$ & 402 & 2009-2011 & & 329 & 333 & 40 & 702 \\
\hline 17 Teresina & $-5,03^{\circ}$ & $-42,80^{\circ}$ & 74 & $2008-2011$ & 332 & 320 & 294 & 72 & 1018 \\
\hline 18 Uruçuí & $-7,47^{\circ}$ & $-44,34^{\circ}$ & 393 & $2008-2011$ & 300 & 362 & 331 & 164 & 1157 \\
\hline 19 Valença do Piauí & $-6,40^{\circ}$ & $-41,74^{\circ}$ & 301 & 2009-2011 & 258 & 332 & 150 & 179 & 919 \\
\hline
\end{tabular}


métodos, determinou-se o coeficiente de correlação (r) linear de Pearson (HOPKINS, 2000). Usaram-se os valores de "r" maiores ou iguais a 0,50 (+r ou -r), que é tida como uma correlação alta, para se fazer a análise de regressão (VANZELA et al., 2007; SOUSA et al., 2010). Para todas as análises usou-se o software SAS (SAS Institute, 2000), com exceção do índice de Willmott (WILLMOTT et al., 1985) e o coeficiente de desempenho ou confiança (CAMARGO, SENTELHAS, 1997), que foram calculados com o auxílio da planilha eletrônica Excel 2007.

\section{Resultados e discussão}

A comparação de médias de ETo pelo teste t é apresentada nas Tabelas 2 e 3. No período seco (Tabela 2), a ETo média diária estimada por TH $(4,54 \pm 0,21 \mathrm{~mm})$ foi superior a todos os demais métodos em todos os municípios avaliados. Apenas em Paulistana a estimativa da ETo por TH não diferiu estatisticamente da estimativa de ETo por PM-FAO, indicando ser um método simplificado de possível utilização nesse município.

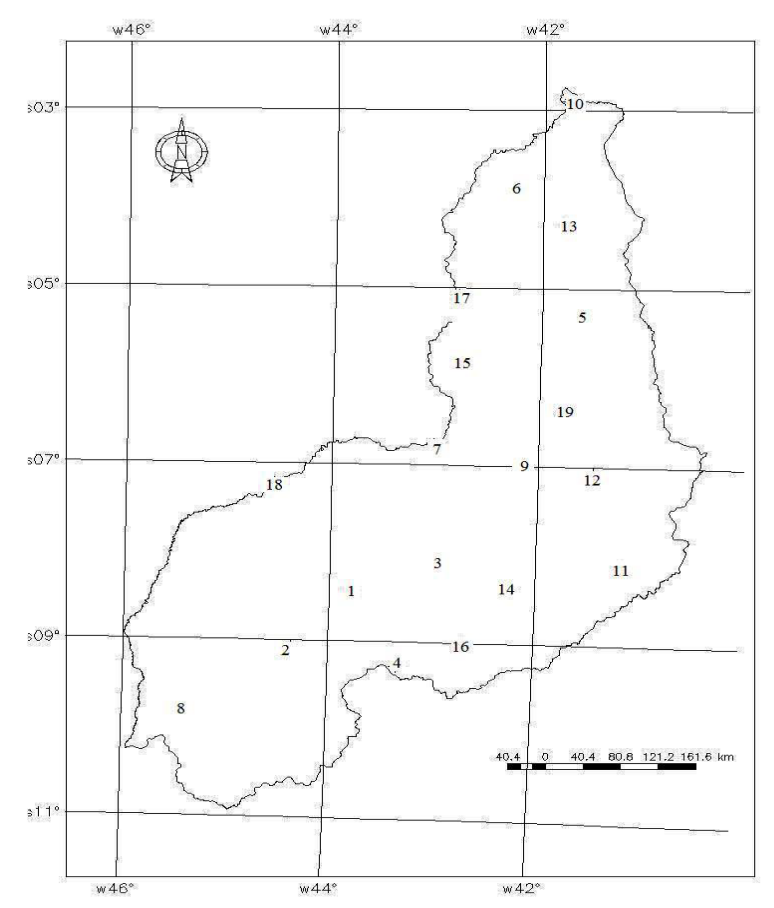

Figura 1. Mapa do Piauí com a posição geográfica das estações meteorológicas automáticas do INMET utilizadas no estudo.

Tabela 2. Comparação de médias diárias de evapotranspiração de referência (ETo, mm) estimada por cada método, no período seco, para as estações meteorológicas utilizadas no estudo.

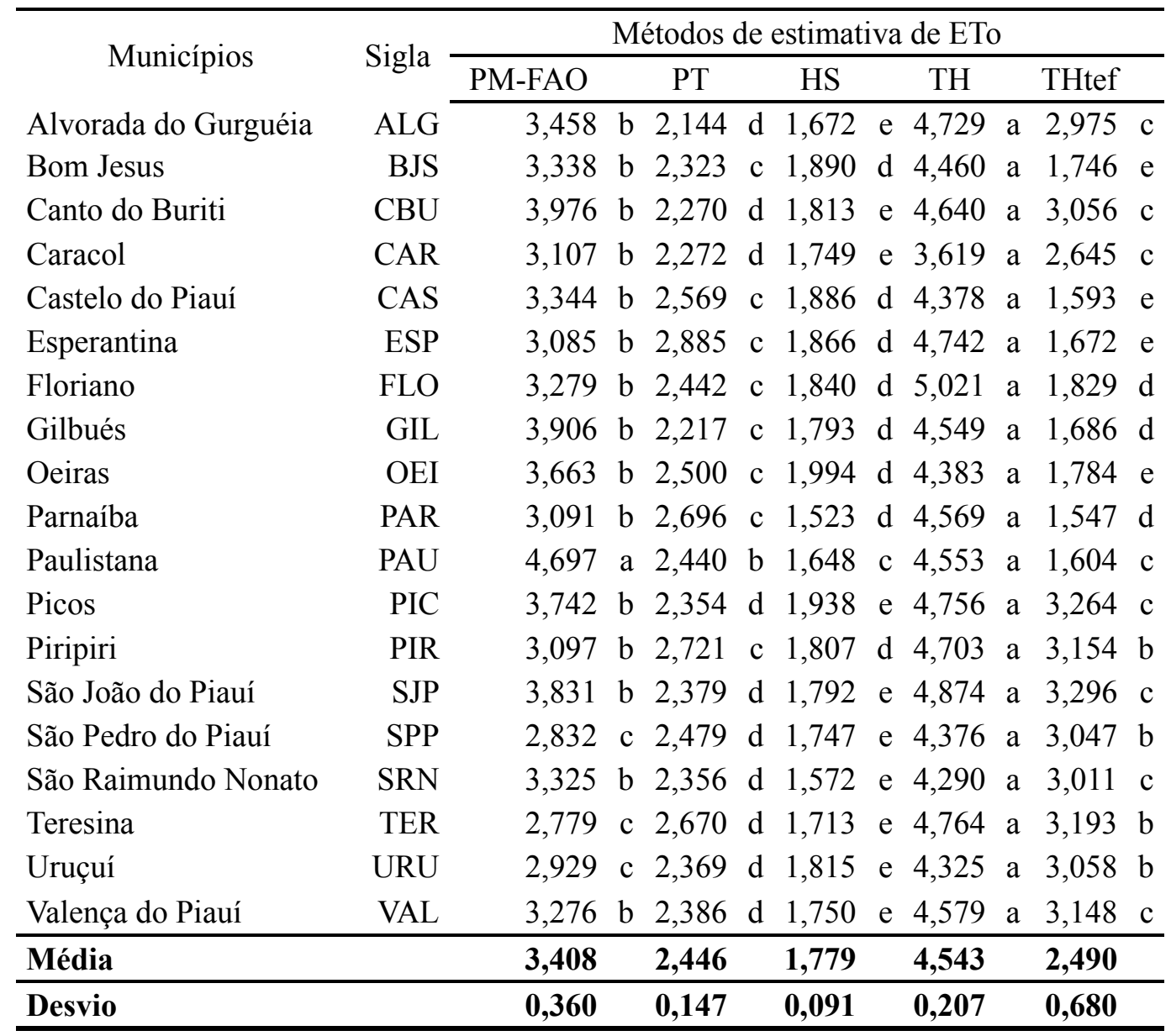

PM-FAO: Penman-Monteith FAO; PT: Priestley-Taylor; HS: Hargreaves \& Samani;

TH: Thornthwaite; THtef: Thornthwaite com temperatura efetiva. Médias seguidas de mesma letra na horie zontal não diferem estatisticamente pelo teste t a $5 \%$ de probabilidade. 
Por outro lado, o método de HS promoveu as menores estimativas diárias de ETo (1,779 $\pm 0,09 \mathrm{~mm})$. Em Piripiri, o método de THtef não diferiu de PM-FAO, demonstrando ser o melhor método simplificado para estimativa da ETo. Essa tendência deveu-se à baixa amplitude térmica encontrada nessa estação, que afeta a estimativa da temperatura efetiva, com relação às demais estações. Embora em Picos, São João do Piauí, São Raimundo Nonato e Valença do Piauí tenha havido diferença entre as estimativas de ETo por THtef em relação a PM-FAO, o desvio médio da estimativa foi da ordem de $10,8 \%(0,364 \mathrm{~mm})$, permitindo indicar a aplicação de THtef para esses municípios. Esses municípios situam-se em região de clima semiárido, condição essa indicada para aplicação do método THtef (CAMARGO et al., 1999). O método de THtef superestimou ETo em relação ao método PM-FAO apenas nos municípios de São Pedro do Piauí, Teresina e Uruçuí, em cerca de 0,238 $\mathrm{mm} \mathrm{dia}^{-1}(7 \%)$, $0,414 \mathrm{~mm} \mathrm{dia}^{-1}(14 \%)$ e $0,119 \mathrm{~mm} \mathrm{dia}{ }^{-1}(4 \%)$, respectivamente (Tabela 2).

O método de PT subestimou PM-FAO em todos os municípios avaliados; porém, por utilizar apenas dados de radiação solar não se distanciou tanto de PM-FAO quanto HS e THtef (Tabela 2). Figueredo Júnior et al. (2005) ressaltam que os métodos que empregam somente a temperatura do ar tendem a limitar as condições climáticas para estimativa da ETo, já que, dependendo das condições de umidade relativa do ar e velocidade do vento, a estimativa de ETo será diferente para os mesmos valores de temperatura do ar.

No período chuvoso (Tabela 3), a ETo média diária esti-

Tabela 3. Comparação de médias diárias de evapotranspiração de referência (ETo, mm) estimada por cada método no período chuvoso, para as estações meteorológicas utilizadas no estudo.

\begin{tabular}{|c|c|c|c|c|c|c|c|c|c|c|}
\hline \multirow{2}{*}{ Municípios } & \multirow{2}{*}{ Sigla } & \multicolumn{9}{|c|}{ Métodos de estimativa de ETo } \\
\hline & & PM-FAO & & $\mathrm{PT}$ & & $\mathrm{HS}$ & & $\mathrm{TH}$ & & THtef \\
\hline Alvorada do Gurguéia & ALG & 3,215 & $\mathrm{~b}$ & 3,138 & $\mathrm{~b}$ & 1,536 & $\mathrm{~d}$ & 4,433 & $\bar{a}$ & 2,754 \\
\hline Bom Jesus & BJS & 2,925 & $\mathrm{c}$ & 3,309 & $\mathrm{~b}$ & 1,649 & $\mathrm{~d}$ & 3,913 & $\mathrm{a}$ & 1,294 \\
\hline Canto do Buriti & $\mathrm{CBU}$ & 3,236 & $\mathrm{~b}$ & 3,427 & $\mathrm{~b}$ & 1,670 & $\mathrm{~d}$ & 3,933 & $\mathrm{a}$ & 2,769 \\
\hline Caracol & CAR & 3,170 & $\mathrm{c}$ & 3,409 & $\mathrm{~b}$ & 1,666 & $\mathrm{e}$ & 3,715 & $\mathrm{a}$ & 2,659 \\
\hline Castelo do Piauí & CAS & 3,609 & $\mathrm{~b}$ & 3,256 & $\mathrm{~b}$ & 1,660 & 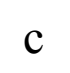 & 4,693 & $\mathrm{a}$ & 1,595 \\
\hline Esperantina & ESP & 3,234 & $\mathrm{c}$ & 3,617 & $\mathrm{~b}$ & 1,691 & $\mathrm{~d}$ & 4,437 & $\mathrm{a}$ & 1,445 \\
\hline Floriano & FLO & 2,812 & $\mathrm{c}$ & 3,222 & $\mathrm{~b}$ & 1,611 & $\mathrm{~d}$ & 4,362 & $\mathrm{a}$ & 1,262 \\
\hline Gilbués & GIL & 3,225 & $\mathrm{c}$ & 3,485 & $\mathrm{~b}$ & 1,643 & $\mathrm{~d}$ & 3,871 & $\mathrm{a}$ & 1,260 \\
\hline Oeiras & OEI & 3,144 & $\mathrm{c}$ & 3,514 & $\mathrm{~b}$ & 1,725 & $\mathrm{~d}$ & 4,383 & $\mathrm{a}$ & 1,377 \\
\hline Parnaíba & PAR & 133 & $\mathrm{~b}$ & 3,210 & $\mathrm{~b}$ & 1,484 & $\mathrm{c}$ & 4,241 & $\mathrm{a}$ & 1,378 \\
\hline Paulistana & PAU & 3,827 & $\mathrm{~b}$ & 3,376 & $\mathrm{c}$ & 1,678 & $\mathrm{~d}$ & 4,469 & $\mathrm{a}$ & 1,482 \\
\hline Picos & PIC & 3,417 & $\mathrm{~b}$ & 3,425 & $\mathrm{~b}$ & 1,787 & $\mathrm{~d}$ & 4,541 & $\mathrm{a}$ & 3,132 \\
\hline Piripiri & PIR & 3,341 & $\mathrm{c}$ & 3,483 & $\mathrm{~b}$ & 1,689 & e & 4,439 & $\mathrm{a}$ & 3,069 \\
\hline São João do Piauí & SJP & 3,328 & $\mathrm{c}$ & 3,501 & $\mathrm{~b}$ & 1,722 & $\mathrm{e}$ & 4,438 & $\mathrm{a}$ & 3,118 \\
\hline São Pedro do Piauí & SPP & 2,935 & $\mathrm{c}$ & 3,372 & $\mathrm{~b}$ & 1,611 & $\mathrm{~d}$ & 3,970 & $\mathrm{a}$ & 2,893 \\
\hline São Raimundo Nonato & SRN & 3,371 & $\mathrm{c}$ & 3,549 & $\mathrm{~b}$ & 1,386 & e & 4,790 & $\mathrm{a}$ & 3,132 \\
\hline Teresina & TER & 2,919 & $d$ & 3,364 & $\mathrm{~b}$ & 1,588 & $\mathrm{e}$ & 4,457 & $\mathrm{a}$ & 3,060 \\
\hline Uruçuí & URU & 2,730 & $\mathrm{c}$ & 3,206 & $\mathrm{~b}$ & 1,597 & 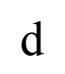 & 3,757 & $\mathrm{a}$ & 2,835 \\
\hline Valença do Piauí & VAL & 3,030 & $\mathrm{c}$ & 3,235 & $\mathrm{~b}$ & 1,675 & $d$ & 4,146 & $\mathrm{a}$ & 2,996 \\
\hline Média $(\mathrm{mm})$ & & 3,190 & & 3,374 & & 1,635 & & 4,263 & & 2,290 \\
\hline Desvio (mm) & & 0,201 & & 0,111 & & 0,067 & & 0,269 & & 0,761 \\
\hline
\end{tabular}

PM-FAO: Penman-Monteith FAO; PT: Priestley-Taylor; HS: Hargreaves \& Samani;

TH: Thornthwaite; THtef: Thornthwaite com temperatura efetiva. Médias seguidas de mesma letra na horizontal não diferem estatistii camente pelo teste $t$ a $5 \%$ de probabilidade. 
mada por TH $(4,26 \pm 0,27 \mathrm{~mm})$ também foi superior a todos os demais métodos em todos os municípios avaliados, diferindo estatisticamente da estimativa de ETo por PM-FAO em todos os locais. Em São Pedro do Piauí, Uruçuí e Valença do Piauí as estimativas de ETo efetuadas pelo método de THtef não diferiram estatisticamente da estimativa de ETo estimada por PM-FAO. O método de PT não diferiu estatisticamente do PM-FAO nos municípios de Alvorada do Gurguéia, Canto do Buriti, Castelo do Piauí, Parnaíba e Picos, o que indica que para esta época do ano (chuvoso) pode-se utilizar o método de PT para a estimativa de ETo quando se dispõem apenas de dados de radiação solar.

Os métodos de PT $(3,37 \pm 0,11 \mathrm{~mm})$ e TH $(4,26 \pm 0,27 \mathrm{~mm})$ se mostraram como métodos que superestimam a ETo em relação ao método de PM-FAO $(3,19 \pm 0,2 \mathrm{~mm})$ em quase todos os municípios, com exceção de Alvorada do Gurguéia, Castelo do Piauí, Parnaíba, Paulistana e Picos. A redução da radiação solar causada pela elevação da nebulosidade nessa época do ano é responsável por essa tendência. Já os métodos de HS $(1,63 \pm 0,07 \mathrm{~mm})$ e THtef $(2,29 \pm 0,76 \mathrm{~mm}) \mathrm{su}$ bestimaram a ETo em relação a PM-FAO, devido à redução da amplitude térmica típica do período chuvoso (MEDEIROS, 2002).

O município de Esperantina apresentou a maior corre- lação entre os métodos de HS $\left(\mathrm{r}^{2}=0,83\right)$, TH $\left(\mathrm{r}^{2}=0,83\right)$ e THtef $\left(r^{2}=0,88\right)$ e o método padrão PM-FAO, indicando que a estimativa de ETo por esses métodos pode ser utilizada em substituição ao método PM-FAO. Em Parnaíba observou-se a maior correlação entre PT e PM-FAO $\left(r^{2}=0,81\right)$. Essa melhor correlação também indica que o método de PT pode ser utilizado em substituição ao método PM-FAO, o que era esperado, já que o método de PT é uma simplificação do método PM-FAO (SILVA et al., 2011). Em Eunápolis-BA e Petrolina-PE, Tagliaferre et al. (2010) e Souza et al. (2011) encontraram correlação de 0,92 e 0,75, respectivamente, entre os métodos de PT e PM-FAO. Back, Rosso (2006) atribuíram essa elevada correlação entre PT e PM-FAO ao fato de serem métodos que utilizam a radiação solar, que é o principal fator meteorológico que controla a evapotranspiração.

As estimativas de ETo por TH e PM-FAO apresentaram correlação variando de "alta" a "quase perfeita" (HOPKINS, 2000), notadamente, no período chuvoso (Tabela 4), indicando a possibilidade de substituição das estimativas de ETo de PM-FAO por TH. Essa tendência não é muito comum na literatura (SALES, 2008; ARAÚJO et al., 2010; BACK, ROSSO, 2006), já que a princípio o método de TH foi concebido para estimativas mensais de ETo ao passo que o mé-

Tabela 4. Análise de regressão linear, com resultados do coeficiente de determinação $\left(\mathrm{r}^{2}\right)$ e o coeficiente angular (b) aplicada aos municípios do Estado do Piauí, tendo os métodos simplificados como variável dependente.

\begin{tabular}{|c|c|c|c|c|c|c|c|c|c|c|c|c|c|c|c|c|}
\hline \multirow{3}{*}{ Sigla } & \multicolumn{4}{|c|}{$\mathrm{HS}$} & \multicolumn{4}{|c|}{$\overline{\mathrm{PT}}$} & \multicolumn{4}{|c|}{$\overline{\mathrm{TH}}$} & \multicolumn{4}{|c|}{ THtef } \\
\hline & \multicolumn{2}{|c|}{ Seco } & \multicolumn{2}{|c|}{ Chuvoso } & \multicolumn{2}{|c|}{ Seco } & \multicolumn{2}{|c|}{ Chuvoso } & \multicolumn{2}{|c|}{ Seco } & \multicolumn{2}{|c|}{ Chuvoso } & \multicolumn{2}{|c|}{ Seco } & \multicolumn{2}{|c|}{ Chuvoso } \\
\hline & $\mathrm{r}^{2}$ & $\mathrm{~b}$ & $\mathrm{r}^{2}$ & B & $\mathrm{r}^{2}$ & $\mathrm{~b}$ & $\mathrm{r}^{2}$ & $\mathrm{~b}$ & $\mathrm{r}^{2}$ & $\mathrm{~b}$ & $\mathrm{r}^{2}$ & $\mathrm{~b}$ & $\mathrm{r}^{2}$ & $\mathrm{~b}$ & $\overline{r^{2}}$ & $\mathrm{~b}$ \\
\hline ALG & 0,38 & 0,47 & 0,50 & 0,46 & $*$ & * & 0,92 & 1,08 & 0,57 & 1,27 & 0,74 & 1,24 & 0,64 & 0,82 & 0,76 & 0,82 \\
\hline BJS & 0,47 & 0,53 & 0,75 & 0,54 & $*$ & $*$ & 0,91 & 1,12 & 0,52 & 1,27 & 0,73 & 1,30 & 0,55 & 0,51 & 0,72 & 0,43 \\
\hline $\mathrm{CBU}$ & 0,26 & 0,40 & 0,62 & 0,50 & $*$ & $*$ & 0,83 & 1,05 & 0,43 & 1,02 & 0,74 & 1,19 & 0,37 & 0,67 & 0,77 & 0,83 \\
\hline CAR & 0,65 & 0,55 & 0,60 & 0,51 & 0,41 & 0,71 & 0,82 & 1,06 & 0,56 & 1,15 & 0,67 & 1,14 & 0,61 & 0,83 & 0,67 & 0,80 \\
\hline CAS & 0,60 & 0,54 & 0,48 & 0,44 & 0,71 & 0,73 & 0,67 & 0,92 & 0,79 & 1,26 & 0,72 & 1,25 & 0,79 & 0,47 & 0,85 & 0,44 \\
\hline ESP & 0,83 & 0,59 & 0,69 & 0,51 & 0,78 & 0,91 & 0,75 & 1,10 & 0,83 & 1,49 & 0,71 & 1,35 & 0,88 & 0,54 & 0,72 & 0,45 \\
\hline FLO & 0,27 & 0,53 & 0,65 & 0,56 & $*$ & $*$ & 0,96 & 1,14 & 0,44 & 1,45 & 0,67 & 1,52 & 0,45 & 0,55 & 0,66 & 0,44 \\
\hline GIL & 0,27 & 0,44 & 0,58 & 0,49 & * & * & 0,87 & 1,07 & 0,34 & 1,11 & 0,70 & 1,17 & 0,32 & 0,42 & 0,74 & 0,38 \\
\hline OEI & 0,34 & 0,51 & 0,66 & 0,54 & $*$ & $*$ & 0,88 & 1,11 & 0,43 & 1,22 & 0,67 & 1,36 & 0,44 & 0,47 & 0,64 & 0,43 \\
\hline PAR & 0,45 & 0,46 & 0,38 & 0,45 & 0,81 & 0,84 & 0,77 & 1,00 & 0,50 & 1,40 & 0,60 & 1,31 & 0,53 & 0,48 & 0,69 & 0,43 \\
\hline PAU & 0,32 & 0,34 & 0,32 & 0,41 & 0,47 & 0,49 & 0,54 & 0,85 & 0,36 & 0,94 & 0,70 & 1,11 & 0,32 & 0,34 & 0,71 & 0,38 \\
\hline PIC & 0,67 & 0,50 & 0,78 & 0,50 & 0,45 & 0,60 & 0,65 & 0,97 & 0,69 & 1,22 & 0,78 & 1,29 & 0,69 & 0,83 & 0,83 & 0,87 \\
\hline PIR & 0,66 & 0,57 & 0,61 & 0,49 & 0,57 & 0,85 & 0,54 & 1,02 & 0,72 & 1,47 & 0,72 & 1,31 & 0,79 & 0,98 & 0,76 & 0,89 \\
\hline SJP & $*$ & $*$ & 0,72 & 0,50 & 0,27 & 0,60 & 0,80 & 1,03 & 0,38 & 1,22 & 0,75 & 1,30 & 0,32 & 0,82 & 0,76 & 0,90 \\
\hline SPP & 0,57 & 0,60 & 0,63 & 0,54 & 0,59 & 0,86 & 0,97 & 1,15 & 0,62 & 1,51 & 0,56 & 1,33 & 0,64 & 1,04 & 0,61 & 0,96 \\
\hline SRN & 0,57 & 0,45 & 0,52 & 0,40 & 0,47 & 0,69 & 0,85 & 1,04 & 0,52 & 1,24 & 0,54 & 1,36 & 0,56 & 0,86 & 0,62 & 0,89 \\
\hline TER & 0,47 & 0,61 & 0,26 & 0,53 & 0,72 & 0,94 & 0,93 & 1,15 & 0,63 & 1,67 & 0,62 & 1,50 & 0,71 & 1,12 & 0,62 & 1,02 \\
\hline URU & 0,60 & 0,59 & 0,67 & 0,56 & * & * & 0,97 & 1,17 & 0,37 & 1,41 & 0,67 & 1,33 & 0,42 & 0,98 & 0,71 & 0,98 \\
\hline VAL & 0,34 & 0,52 & 0,60 & 0,54 & 0,28 & 0,70 & 0,77 & 1,05 & 0,46 & 1,35 & 0,66 & 1,34 & 0,41 & 0,92 & 0,69 & 0,95 \\
\hline
\end{tabular}

*Estações que não apresentaram valores de $\mathrm{r}^{2}$ e b mostraram valores de correlação (r) abaixo de 0,50. 
todo de PM-FAO é fisicamente mais consistente e utilizado para estimativas diárias de ETo (AZEVEDO, 1999). A estimativa de ETo por métodos que utilizam apenas dados de temperatura do ar tendem a limitar a representatividade das condições climáticas, pois com os mesmos valores de temperatura do ar pode-se ter demandas hídricas atmosféricas diferentes, dependendo das condições de umidade relativa do ar e do vento (CONCEIÇÃO, 2003). Por outro lado, é um resultado muito interessante, pois apenas com dados de temperatura média do ar pode-se estimar a ETo com a mesma confiabilidade e qualidade que seria com a adoção do método PM-FAO.

Comparando-se o método de THtef com o de TH, houve um acréscimo nos valores de $\mathrm{r}^{2}$, para quase todos os locais. Esse comportamento indica que a adoção de valores de temperatura efetiva do ar em substituição à temperatura média mensal do ar na estimativa de ETo acrescentou melhoria na estimativa de ETo, devendo, ser indicada a sua adoção. De fato, a adaptação proposta por Camargo et al. (1999) na estimativa da ETo por TH, deveria trazer melhores resultados em termos de estimativa de ETo em regiões com clima muito úmido ou árido.
Quanto a avaliação do desempenho (Tabelas 5 e 6), durante o período seco do ano, o método de HS apresentou-se com desempenho variando de "mau" a "péssimo". Para nenhum dos municípios obteve-se índice de confiança ou desempenho aceitável para a estimativa de ETo padrão. Tendência semelhante foi observada por Araújo et al. (2007), que classificou como "sofrível" o desempenho do método de HS em comparação a PM-FAO ao avaliar os dados mensais de ETo de Boa Vista, RR.

Os municípios de Esperantina, Parnaíba e Teresina apresentaram desempenho classificado como "bom" para o método de PT, demonstrando que para estes municípios eles pode-se utilizar o método PT para estimativas de ETo em substituição ao método padrão (PM-FAO). Isso pode ter ocorrido pela influência climática do Estado do Maranhão, pois os municípios estão localizados na fronteira com o aquele Estado do Maranhão e próximo à linha do Equador, com alta incidência de radiação solar e valores mais amenos de temperatura do ar. Reis et al. (2007), em estudo comparativo de métodos de estimativa de ETo, para municípios do Espírito Santo, observaram que para todos os municípios, o método de PT teve correlação variando de

Tabela 5. Parâmetros estatísticos de índice de confiança (c), índice de Willmott (d), coeficiente de correlação (r) e classificação do desempenho (D) para os métodos simplificados de Hargreaves \& Samani (HS) e Priestley-Taylor (PT) em relação a Penman-Monteith FAO, em escala diária, aplicado aos municípios avaliados.

\begin{tabular}{|c|c|c|c|c|c|c|c|c|c|c|c|c|c|c|c|c|}
\hline \multirow{3}{*}{ Sigla } & \multicolumn{8}{|c|}{ HS } & \multicolumn{8}{|c|}{ PT } \\
\hline & \multicolumn{4}{|c|}{ Seco } & \multicolumn{4}{|c|}{ Chuvoso } & \multicolumn{4}{|c|}{ Seco } & \multicolumn{4}{|c|}{ Chuvoso } \\
\hline & d & $\mathrm{r}$ & $\mathrm{c}$ & $\mathrm{D}$ & $\mathrm{d}$ & $\mathrm{r}$ & $\mathrm{c}$ & $\mathrm{D}$ & $\mathrm{d}$ & $\mathrm{r}$ & $\mathrm{c}$ & $\mathrm{D}$ & d & r & $\mathrm{c}$ & $\mathrm{D}$ \\
\hline$\overline{A L G}$ & 0,45 & 0,62 & 0,28 & $\mathrm{Pe}$ & 0,45 & 0,70 & 0,32 & $\mathrm{Pe}$ & 0,50 & 0,48 & 0,24 & $\mathrm{Pe}$ & 0,97 & 0,96 & 0,93 & $\overline{\mathrm{Ot}}$ \\
\hline BJS & 0,52 & 0,69 & 0,36 & $\mathrm{Pe}$ & 0,92 & 0,86 & 0,80 & $\mathrm{MB}$ & 0,52 & 0,39 & 0,20 & $\mathrm{Pe}$ & 0,92 & 0,95 & 0,88 & Ot \\
\hline $\mathrm{CBU}$ & 0,40 & 0,51 & 0,20 & $\mathrm{Pe}$ & 0,26 & 0,79 & 0,21 & $\mathrm{Pe}$ & 0,43 & 0,39 & 0,17 & $\mathrm{Pe}$ & 0,41 & 0,91 & 0,37 & $\mathrm{Pe}$ \\
\hline CAR & 0,52 & 0,81 & 0,42 & $\mathrm{Ma}$ & 0,47 & 0,77 & 0,37 & $\mathrm{Pe}$ & 0,64 & 0,64 & 0,41 & $\mathrm{Ma}$ & 0,93 & 0,90 & 0,84 & MB \\
\hline CAS & 0,53 & 0,78 & 0,42 & $\mathrm{Ma}$ & 0,45 & 0,70 & 0,32 & $\mathrm{Pe}$ & 0,64 & 0,84 & 0,54 & So & 0,86 & 0,82 & 0,71 & $\mathrm{Bm}$ \\
\hline ESP & 0,55 & 0,91 & 0,50 & $\mathrm{Ma}$ & 0,43 & 0,83 & 0,36 & $\mathrm{Pe}$ & 0,81 & 0,88 & 0,71 & $\mathrm{Bm}$ & 0,85 & 0,87 & 0,74 & $\mathrm{Bm}$ \\
\hline FLO & 0,48 & 0,52 & 0,25 & $\mathrm{Pe}$ & 0,47 & 0,81 & 0,38 & $\mathrm{Pe}$ & 0,52 & 0,31 & 0,16 & $\mathrm{Pe}$ & 0,90 & 0,98 & 0,89 & Ot \\
\hline GIL & 0,42 & 0,52 & 0,22 & $\mathrm{Pe}$ & 0,44 & 0,76 & 0,33 & $\mathrm{Pe}$ & 0,43 & 0,19 & 0,08 & $\mathrm{Pe}$ & 0,94 & 0,93 & 0,88 & Ot \\
\hline OEI & 0,50 & 0,58 & 0,29 & $\mathrm{Pe}$ & 0,46 & 0,81 & 0,37 & $\mathrm{Pe}$ & 0,51 & 0,36 & 0,19 & $\mathrm{Pe}$ & 0,91 & 0,94 & 0,85 & $\mathrm{MB}$ \\
\hline PAR & 0,46 & 0,67 & 0,31 & $\mathrm{Pe}$ & 0,44 & 0,62 & 0,27 & $\mathrm{Pe}$ & 0,78 & 0,90 & 0,70 & $\mathrm{Bm}$ & 0,92 & 0,88 & 0,80 & $\mathrm{MB}$ \\
\hline PAU & 0,38 & 0,57 & 0,21 & $\mathrm{Pe}$ & 0,43 & 0,57 & 0,25 & $\mathrm{Pe}$ & 0,45 & 0,69 & 0,31 & $\mathrm{Pe}$ & 0,73 & 0,74 & 0,54 & So \\
\hline PIC & 0,50 & 0,82 & 0,41 & $\mathrm{Ma}$ & 0,49 & 0,88 & 0,43 & $\mathrm{Ma}$ & 0,53 & 0,67 & 0,36 & $\mathrm{Pe}$ & 0,87 & 0,80 & 0,70 & $\mathrm{Bm}$ \\
\hline PIR & 0,51 & 0,81 & 0,41 & $\mathrm{Ma}$ & 0,43 & 0,78 & 0,34 & $\mathrm{Pe}$ & 0,69 & 0,75 & 0,52 & So & 0,82 & 0,74 & 0,61 & $\mathrm{Md}$ \\
\hline SJP & 0,42 & 0,48 & 0,20 & $\mathrm{Pe}$ & 0,46 & 0,85 & 0,39 & $\mathrm{Pe}$ & 0,49 & 0,52 & 0,25 & $\mathrm{Pe}$ & 0,93 & 0,89 & 0,83 & $\mathrm{MB}$ \\
\hline SPP & 0,49 & 0,75 & 0,37 & $\mathrm{Pe}$ & 0,41 & 0,79 & 0,33 & $\mathrm{Pe}$ & 0,76 & 0,77 & 0,58 & So & 0,88 & 0,98 & 0,86 & Ot \\
\hline SRN & 0,47 & 0,76 & 0,36 & $\mathrm{Pe}$ & 0,40 & 0,72 & 0,29 & $\mathrm{Pe}$ & 0,63 & 0,69 & 0,43 & $\mathrm{Ma}$ & 0,94 & 0,92 & 0,87 & $\mathrm{Ot}$ \\
\hline TER & 0,50 & 0,68 & 0,34 & $\mathrm{Pe}$ & 0,40 & 0,51 & 0,21 & $\mathrm{Pe}$ & 0,85 & 0,85 & 0,72 & $\mathrm{Bm}$ & 0,86 & 0,96 & 0,83 & $\mathrm{MB}$ \\
\hline URU & 0,53 & 0,77 & 0,41 & $\mathrm{Ma}$ & 0,51 & 0,82 & 0,42 & $\mathrm{Ma}$ & 0,59 & 0,48 & 0,28 & $\mathrm{Pe}$ & 0,91 & 0,99 & 0,89 & $\mathrm{Ot}$ \\
\hline VAL & 0,44 & 0,58 & 0,26 & $\mathrm{Pe}$ & 0,45 & 0,78 & 0,35 & $\mathrm{Pe}$ & 0,53 & 0,53 & 0,28 & $\mathrm{Pe}$ & 0,89 & 0,88 & 0,78 & MB \\
\hline
\end{tabular}

Ot = ótimo; $\mathrm{MB}=$ muito bom; $\mathrm{Bm}=$ bom; $\mathrm{Md}$ = mediano; $\mathrm{So}=$ sofrível; $\mathrm{Ma}=$ mau e $\mathrm{Pe}=$ péssimo. 
"mediano" a "bom" com o método de PM-FAO, para dados diários no período seco.

O desempenho do método de TH com relação a PM-FAO obteve classificação variando de "bom" a "mau". o melhor desempenho foi apresentado apenas em Castelo do Piauí classificado como "bom", os demais municípios variaram a classificação de "mau" a "sofrível". Syperreck (2006) encontrou desempenho "mau" para os métodos de TH e HS em seu estudo para a região de Palotina - PR. Quando se ajustou o método TH com a temperatura efetiva (THtef) observou-se que o melhor índice de confiança ocorreu no município de Piripiri classificado com "mediano", os demais variaram entre "péssimo" e "sofrível".

Durante o período chuvoso, apenas o município de Bom Jesus apresentou melhor desempenho classificado como "muito bom" para HS em comparação a PM-FAO. Sousa et al. (2010) encontraram resultados semelhantes para perímetros irrigados em Sergipe, com índice de desempenho classificado como "muito bom". Oliveira et al. (2010) encontraram desempenho "bom" para o método HS aplicado à região norte da Bahia.
O método de PT apresentou ótimo desempenho em todos os municípios variando de "bom" a "ótimo", com exceção de Canto do Buriti e Paulistana, onde se observou um desempenho "péssimo" (c=0,37) e "sofrível" ( $c=0,54)$, respectivamente. Souza et al. (2011) não encontraram resultados satisfatórios quando analisaram o desempenho do método PT com relação ao PM-FAO, para Petrolina-PE, com índice de confiança classificado como "mau" $(c=0,47)$. Embora com desempenho não satisfatório, o método de PT foi o que apresentou melhor desempenho dentre os métodos empíricos analisados durante o período seco.

Quanto ao método de TH, o município de Paulistana apresentou desempenho "bom" $(c=0,68)$, indicando assim, confiança nas estimativas de ETo no período chuvoso. Medeiros (2002), analisando as estimativas de ETo por TH para Paraipaba - CE, encontrou um desempenho "sofrível". Resultados semelhantes foram observados nos municípios de Bom Jesus, Castelo do Piauí, Parnaíba, Piripiri, São João do Piauí e Uruçuí. Mandelli, Conceição (2005) ao analisarem as estimativas de ETo por seis métodos empíricos comparando com PM-FAO, para Bento Gonçalves-RS, concluíram

Tabela 6. Parâmetros estatísticos de índice de confiança (c), índice de Willmott (d), coeficiente de correlação (r) e classificação do desempenho (D) para os métodos simplificados de Thornthwaite (TH) e Thornthwaite com temperatura efetiva (THtef) em relação a Penman-Monteith FAO, em escala diária, aplicados aos municípios avaliados.

\begin{tabular}{|c|c|c|c|c|c|c|c|c|c|c|c|c|c|c|c|c|}
\hline \multirow{3}{*}{ Sigla } & \multicolumn{8}{|c|}{$\mathrm{TH}$} & \multicolumn{8}{|c|}{ THtef } \\
\hline & \multicolumn{4}{|c|}{ Seco } & \multicolumn{4}{|c|}{ Chuvoso } & \multicolumn{4}{|c|}{ Seco } & \multicolumn{4}{|c|}{ Chuvoso } \\
\hline & $\mathrm{d}$ & $\mathrm{r}$ & $\mathrm{C}$ & $\mathrm{D}$ & $\mathrm{d}$ & $r$ & $\mathrm{c}$ & $\mathrm{D}$ & $\mathrm{d}$ & $\mathrm{r}$ & $\mathrm{c}$ & $\mathrm{D}$ & $\mathrm{d}$ & $\mathrm{r}$ & $\mathrm{c}$ & $\mathrm{D}$ \\
\hline$\overline{\mathrm{ALG}}$ & 0.60 & 0.75 & 0.45 & $\mathrm{Ma}$ & 0.56 & 0.86 & 0.48 & $\mathrm{Ma}$ & 0.67 & 0.80 & 0.54 & So & 0.98 & 0.87 & 0.86 & $\mathrm{Ot}$ \\
\hline BJS & 0.68 & 0.72 & 0.49 & $\mathrm{Ma}$ & 0.67 & 0.85 & 0.57 & So & 0.54 & 0.74 & 0.40 & $\mathrm{Pe}$ & 0.45 & 0.85 & 0.39 & $\mathrm{Pe}$ \\
\hline $\mathrm{CBU}$ & 0.74 & 0.66 & 0.49 & $\mathrm{Ma}$ & 0.34 & 0.86 & 0.29 & $\mathrm{Pe}$ & 0.52 & 0.61 & 0.32 & $\mathrm{Pe}$ & 0.35 & 0.88 & 0.30 & $\mathrm{Pe}$ \\
\hline CAR & 0.79 & 0.75 & 0.59 & So & 0.79 & 0.82 & 0.65 & $\mathrm{Md}$ & 0.70 & 0.78 & 0.54 & So & 0.63 & 0.82 & 0.51 & So \\
\hline CAS & 0.74 & 0.89 & 0.66 & $\mathrm{Bm}$ & 0.71 & 0.85 & 0.60 & So & 0.53 & 0.89 & 0.47 & $\mathrm{Ma}$ & 0.49 & 0.92 & 0.45 & $\mathrm{Ma}$ \\
\hline ESP & 0.52 & 0.91 & 0.47 & $\mathrm{Ma}$ & 0.58 & 0.84 & 0.49 & $\mathrm{Ma}$ & 0.54 & 0.94 & 0.51 & So & 0.42 & 0.85 & 0.35 & $\mathrm{Pe}$ \\
\hline FLO & 0.49 & 0.66 & 0.32 & $\mathrm{Ma}$ & 0.44 & 0.82 & 0.36 & $\mathrm{Pe}$ & 0.53 & 0.67 & 0.35 & $\mathrm{Pe}$ & 0.42 & 0.81 & 0.34 & $\mathrm{Pe}$ \\
\hline GIL & 0.66 & 0.59 & 0.39 & $\mathrm{Ma}$ & 0.74 & 0.84 & 0.62 & $\mathrm{Md}$ & 0.43 & 0.57 & 0.24 & $\mathrm{Pe}$ & 0.40 & 0.86 & 0.34 & $\mathrm{Pe}$ \\
\hline OEI & 0.64 & 0.65 & 0.42 & $\mathrm{Ma}$ & 0.54 & 0.82 & 0.45 & $\mathrm{Ma}$ & 0.51 & 0.66 & 0.34 & $\mathrm{Pe}$ & 0.41 & 0.80 & 0.33 & $\mathrm{Pe}$ \\
\hline PAR & 0.56 & 0.71 & 0.40 & $\mathrm{Ma}$ & 0.66 & 0.77 & 0.51 & So & 0.50 & 0.73 & 0.36 & $\mathrm{Pe}$ & 0.47 & 0.83 & 0.39 & $\mathrm{Pe}$ \\
\hline PAU & 0.75 & 0.60 & 0.45 & $\mathrm{Ma}$ & 0.81 & 0.84 & 0.68 & $\mathrm{Bm}$ & 0.39 & 0.57 & 0.22 & $\mathrm{Pe}$ & 0.45 & 0.84 & 0.38 & $\mathrm{Pe}$ \\
\hline PIC & 0.73 & 0.83 & 0.60 & So & 0.70 & 0.89 & 0.62 & $\mathrm{Md}$ & 0.68 & 0.83 & 0.57 & So & 0.72 & 0.91 & 0.65 & $\mathrm{Md}$ \\
\hline PIR & 0.51 & 0.85 & 0.43 & $\mathrm{Ma}$ & 0.65 & 0.85 & 0.56 & So & 0.73 & 0.89 & 0.65 & $\mathrm{Md}$ & 0.73 & 0.87 & 0.64 & $\mathrm{Md}$ \\
\hline SJP & 0.61 & 0.62 & 0.38 & $\mathrm{Ma}$ & 0.67 & 0.86 & 0.58 & So & 0.59 & 0.57 & 0.33 & $\mathrm{Pe}$ & 0.70 & 0.87 & 0.61 & $\mathrm{Md}$ \\
\hline SPP & 0.47 & 0.78 & 0.37 & $\mathrm{Ma}$ & 0.52 & 0.75 & 0.39 & $\mathrm{Pe}$ & 0.71 & 0.80 & 0.57 & So & 0.63 & 0.78 & 0.49 & $\mathrm{Ma}$ \\
\hline SRN & 0.69 & 0.72 & 0.49 & $\mathrm{Ma}$ & 0.50 & 0.73 & 0.37 & $\mathrm{Pe}$ & 0.64 & 0.75 & 0.48 & $\mathrm{Ma}$ & 0.54 & 0.79 & 0.43 & $\mathrm{Ma}$ \\
\hline TER & 0.39 & 0.79 & 0.31 & $\mathrm{Ma}$ & 0.43 & 0.79 & 0.34 & $\mathrm{Pe}$ & 0.70 & 0.84 & 0.59 & So & 0.66 & 0.79 & 0.52 & So \\
\hline URU & 0.52 & 0.61 & 0.32 & $\mathrm{Ma}$ & 0.63 & 0.82 & 0.51 & So & 0.61 & 0.65 & 0.40 & $\mathrm{Pe}$ & 0.63 & 0.84 & 0.53 & So \\
\hline VAL & 0.52 & 0.68 & 0.35 & $\mathrm{Ma}$ & 0.59 & 0.81 & 0.48 & $\mathrm{Ma}$ & 0.63 & 0.64 & 0.40 & $\mathrm{Pe}$ & 0.71 & 0.83 & 0.59 & So \\
\hline
\end{tabular}

Ot = ótimo; $\mathrm{MB}=$ muito bom; $\mathrm{Bm}$ = bom; $\mathrm{Md}$ = mediano; $\mathrm{So}=$ sofrível; $\mathrm{Ma}=$ mau e $\mathrm{Pe}=$ péssimo . 
que, os métodos cujas estimativas são baseadas em dados de radiação solar apresentam melhor desempenho que os métodos baseados em temperatura do ar.

Quanto ao método THtef observou-se desempenho de "ótimo" a "péssimo". Esse resultado foi determinado pelo baixo índice de concordância, pois os índices de precisão apresentaram altos valores variando de "alta" $(r=0,78)$ a "quase perfeita" $(r=0,92)$. O melhor índice de confiança foi encontrado em Alvorada do Gurguéia com $c=0,86$, para este município sendo recomendado o uso do método de THtef para se estimar ETo. Back (2008) ao analisar o período chuvoso de Urussanga-SC, numa escala decendial, encontrou um desempenho "bom" para o método THtef. Lima (2005) recomenda o uso do método da amplitude de temperatura quando não se dispõe de dados de radiação solar.

\section{Conclusões}

A ETo média diária estimada por TH foi superior a todos os demais métodos em todos os municípios avaliados, diferindo estatisticamente da estimativa de ETo por PM-FAO, nos períodos seco e chuvoso. Por outro lado, o método de HS promoveu as menores estimativas diárias de ETo.

O método de PT apresentou desempenho de "bom" a "ótimo" em quase todos os municípios avaliados, notadamente, no período chuvoso. Por outro lado, o método de TH não apresentou desempenho satisfatório com relação a PM-FAO. A adoção da correção da estimativa de ETo por TH pelo emprego da temperatura efetiva (THtef) eleva o nível de desempenho das estimativas de ETo com relação a PM-FAO.

\section{Referências}

ALLEN, R.G.; PEREIRA, L.S.; RAES, D.; SMITH, M. Crop evapotranspiration: guidelines for computing crop water requirements. Rome: FAO, 1998. (Irrigation and Drainage Paper, n. 56).

ARAÚJO, E. M.; OLIVEIRA, J. B.; ARAÚJO, E. M.; LÊDO, E. R.; SILVA, M. G. Desempenho de métodos de estimativa de ETo correlacionados com a equação padrão Penman-Monteith FAO 56, em cidades do Estado do Ceará. Revista ACTA Tecnológica, v. 5, n. 2, 2010.

ARAÚJO, W. F.; COSTA, S. A. A.; SANTOS, A. E. Comparação entre métodos de estimativa da Evapotranspiração de referência (ETo) para Boa VistaRR. Revista Caatinga, Mossoró, RN, v.20, n.4, p.84-88, 2007.

AZEVEDO, B.M. Evapotranspiração de referência obtida com a Razão de Bowen, Lisímetro de pesagem e equação de Penman-Monteith utilizando sistemas automáticos. Tese (Doutorado em Irrigação e Drenagem) - Escola Superior de Agricultura "Luiz de Queiroz", Universidade de São Paulo, Piracicaba, 81 p., 1999.

BACK, A.J. Desempenho de métodos empíricos baseados na temperatura do ar para a estimativa da evapotranspiração de referência em Urussanga, SC. Revista Irriga, Botucatu, v.13, n.4, p. 449-466, 2008.

BACK, A.J.; ROSSO, J.C. Comparação de métodos de estimativa da evapotranspiração de referência decendial e mensal em UrussangaSC. In: XIV CONGRESSO BRASILEIRO DE METEOROLOGIA. Anais... Florianópolis: SBMET, 2006. CD-ROM
CAMARGO, A.P.; MARIN, F.R.; SENTELHAS, P.C.; PICINI, A.G. Ajuste da equação de Thornthwaite para estimar a evapotranspiração potencial em climas áridos e super-úmidos, com base na amplitude térmica diária. Revista Brasileira de Agrometeorologia, Santa Maria, v.7, n.2, p. 251-257, 1999.

CAMARGO, A.P.; SENTELHAS, P.C. Avaliação do desempenho de diferentes métodos de estimativa da evapotranspiração potencial no Estado de São Paulo. Revista Brasileira de Agrometeorologia, Santa Maria, RS, v.5, n.1, p.89-97, 1997.

CONCEIÇÃO, M.A.F. Estimativa da evapotranspiração de referência com base na temperatura do ar para as condições do Baixo Rio Grande, SP. Revista Brasileira de Agrometeorologia, Santa Maria, v. 11, n. 2, p. 229-236, 2003.

FIGUEREDO JÚNIOR, L.G.M.; ARAUJO, M.J.V.; ANDRADE JÚNIOR, A.S. Estimativa da evapotranspiração de referência na escala mensal com os métodos de Penman-Monteith, Thornthwaite e PriestleyTaylor para Parnaíba, Piauí. In: XIX CONGRESSO BRASILEIRO DE AGROMETEOROLOGIA, 2005, Campinas. Anais ... Campinas: SBAGRO, 2005. CD-ROM.

HARGREAVES, G.H.; SAMANI, Z.A. Reference crop evapotranspiration from temperature. Applied Engineering Agriculture, v.1, n.2, p. 96-99, 1985.

HOPKINS, W.G. A New View of Statistics: Correlation coefficient. Disponível em http://www.sportsci.org/resource/stats/correl.html. Acesso em 22 ago. 2000.

LIMA, E.P. Evapotranspiração de referência de Penman-Monteith, padrão FAO (1998), a partir de dados de temperatura máxima e mínima de Minas Gerais. Tese (Doutorado em Meteorologia Agrícola), Universidade Federal de Viçosa, 85p., 2005.

MANDELLI, F.; CONCEIÇÃO, M.A.F. Comparação entre métodos de estimativa da evapotranspiração de referência em Bento Gonçalves, RS. Revista Brasileira de Agrometeorologia, Santa Maria, v. 13, n. 2, p. 303-307, 2005.

MEDEIROS, A.T. Estimativa da evapotranspiração de referência a partir da equação de Penman-Monteith, de medidas lisimétricas e de equações empíricas em Paraipaba, CE. Tese (Doutorado em Agronomia) - Escola Superior Luiz de Queiroz, Piracicaba, 103 p., 2002.

OLIVEIRA, G.M.; LEITÃO, M.M.V.B.R.; BISPO, R.C.; SANTOS, I.M.S.; ALMEIDA, A.C. Comparação entre métodos de estimativa da evapotranspiração de referência na região norte da Bahia. Revista Brasileira de Agricultura Irrigada, v.4, n.2, p.104-109, 2010.

PRIESTLEY, C.H.B.; TAYLOR, R.J. On the assessment of surface heat flux and evaporation using large-scale parameters. Monthly Weather Review, v.100, p.81-92, 1972.

REIS, E.F.; BRAGANÇA, R.; GARCIA, G.O.; PEZZOPANE, J.E.M.; TAGLIAFERRE, C. Estudo comparativo da estimativa da evapotranspiração de referência para três localidades do Estado do Espírito Santo no período seco. Idesia, Chile, v. 25, n. 3, p. 75-84, 2007.

SAS INSTITUTE Inc. SAS/STAT: User's Guide. Version 6, ed. 4, v. 1, Cory, NC: SAS Institute Inc., 2000. 943p.

SALES, J. C. Caracterização climática e comparação de métodos de estimativa de evapotranspiração de referência para regiões do estado do Ceará. Tese (Doutorado em Agronomia) - Faculdade de Ciências Agronômicas, Universidade Estadual Paulista "Júlio de Mesquita Filho", 212p., 2008.

SILVA, V. J.; CARVALHO, H. P.; DA SILVA, C. R.; CAMARGO, R.; TEODORO, R. E. F. Desempenho de diferentes métodos de estimativa da evapotranspiração de referência diária em Uberlândia, MG. Bioscience Journal, Uberlândia, v. 27, n. 1, p. 95-101, 2011.

SYPERRECK, V.L.G. Avaliação do desempenho de modelos para estimativa da evapotranspiração de referência para a região de Palotina PR. Dissertação (Mestrado em Agronomia) - Universidade Estadual do Oeste do Paraná, Marechal Cândido Rondon, 48p., 2006.

SOUSA, I.F.; SILVA, V.P.R.; SABINO, F.G.; AGUIAR NETTO, A.O.; SILVA, B. K.N.; AZEVEDO, P.V. Evapotranspiração de referência nos perímetros irrigados do Estado de Sergipe. Revista Brasileira de Engenharia Agrícola e Ambiental, v.14, n.6, p.633-644, 2010. 
SOUZA, L.S.B.; MOURA, M.S.B.; SILVA, T.G.F.; SEDIYAMA, G.C. Comparação de métodos de estimativa da evapotranspiração de referência em Petrolina-PE. In. XVII CONGRESSO BRASILEIRO DE AGROMETEOROLOGIA, Guarapari, 2011. Anais... Guarapari: SBAGRO, 2011. CD-ROM.

TAGLIAFERRE, C.; SILVA, R.A.J.; ROCHA, F.A.; SANTOS, L.C.; SILVA, C.S. Estudo comparativo de diferentes metodologias para determinação da evapotranspiração de referência em Eunápolis-BA. Revista Caatinga, Mossoró, v. 23, n. 1, p. 103-111, 2010.

THORNTHWAITE, C.W. An approach toward a rational classification of climate. Geographical Review, v.38, n.1, p.55-94, 1948.
VANZELA, L.S.; HERNANDEZ, F.B.T.; FERREIRA, E.J.S. Desempenho da estimativa da evapotranspiração de referência em Ilha Solteira - SP. In: XXXVI CONGRESSO BRASILEIRO DE ENGENHARIA AGRÍCOLA, 2007, Bonito, Anais... Bonito: SBEA, 2007.CD-ROM.

WILLMOTT, C.J.; ACKLESON, S. G.; DAVIS, R. E.; FEDDEMA, J. J.; KLINK, K. M.; LEGATES, D. R.; O'DONNELL, J.; ROWE, C. M. Statistics for the evaluation and comparison of models. Journal of Geophysical Research, v. 90, n. C5, p. 8995-9005, 1985.

REFERENCIAÇÃO $\quad$ ANDRADE JUNIOR, A. S. de.; SILVA, C. O. da; SOUSA, V. F. de; RIBEIRO, V. Q. Avaliação de métodos para estimativa da evapotranspiração de referência no estado do Piauí. Agrometeoros, Passo Fundo, v.25, n.1, p.181-190, 2017.

Declaração: os trabalhos estão sendo publicados nesse número de AGROMETEOROS (v.25, n.1, ago 2017) conforme foram aceitos pelo XX Congresso Brasileiro de Agrometeorologia, realizado de 14 a 18 de agosto de 2017, em Juazeiro, BA e Petrolina, PE, sem revisão editorial adicional da revista. 


\title{
Evaluation of methods for estimating reference evapotranspiration in Piaui State, Brazil
}

\author{
Aderson Soares de Andrade Junior ${ }^{1(*)}$, Clescy Oliveira da Silva ${ }^{2}$, Valdemício Ferreira de Sousa ${ }^{3}$, Valdenir Queiroz Ribeiro ${ }^{4}$ \\ ${ }^{1}$ Pesquisador, Embrapa Meio-Norte, Teresina, PI, aderson.andrade@embrapa.br \\ ${ }^{2}$ Mestre em Engenharia Agrícola, UFC, Fortaleza, CE, clescy@gmail.com \\ ${ }^{3}$ Pesquisador, Embrapa Meio-Norte, Teresina, PI, valdemicio.sousa@embrapa.br \\ ${ }^{4}$ Pesquisador, Embrapa Meio-Norte, Teresina, PI, valdenir.queiroz@embrapa.br \\ ${ }^{(*)}$ Corresponding author
}

\section{ARTICLE INFO}

\section{Article history:}

Received 16 June 2017

Accepted 10 August 2017

\section{Index terms:}

empirical equations

confidence index

willmott Index

\section{ABSTRACT}

This study aimed to estimate the reference evapotranspiration (ETo) by methods that use a lower number of meteorological variables, comparing them with the standard Penman-Monteith method. The meteorological data were provided by the National Institute of Meteorology (INMET) of 19 meteorological stations located in the State of Piauí. The estimates of ETo were made for the dry and rainy periods of the year by the methods of Hargreaves \& Samani (HS), Priestley-Taylor (PT), Thornthwaite (TH), and Thornthwaite with effective temperature (THtef) and Penman-Monteith FAO (PM-FAO). The study was modeled using the completely randomized design for each meteorological station. The statistical methods used in the analysis were test " $t$ at the 5\% probability level (for ETo means), Pearson's correlation, linear regression, Willmott's coefficient and confidence index. The PT method presented performance from "good" to "optimum" in almost all municipalities evaluated, notably in the rainy season. The TH method did not perform satisfactorily in relation to PM-FAO. The adoption of the correction of the ETo estimate by $\mathrm{TH}$ by the use of the effective temperature (THtef) raises the performance level of ETo estimates in relation to PM-FAO.

Disclaimer: papers are published in this issue of AGROMETEOROS (v. 25, n.1, aug 2017) as accepted by the XX Congresso Brasileiro de Agrometeorologia, held August 14-18, 2017 in Juazeiro, Bahia and Petrolina, Pernambuco, Brazil, without further revision by editorial board. 